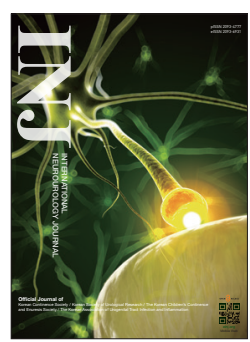

\title{
On Impact Factor
}

\author{
Khae Hawn Kim (iD https://orcid.org/0000-0002-7045-8004 \\ Department of Urology, Gachon University Gil Medical Center, Gachon University of School of Medicine, Incheon, Korea \\ E-mail:kimcho99@gilhospital.com
}

It is a fact universal accepted that competing for a limited position ceding out the top positions principally falls to a particular quantitative parameter. While citation indices may take on such a role, several subjective and personal elements apart from the quality of the science may factor into its being, such as peer evaluation and impact factor of the published journal. In general, when allocating limited resources, such as national or institutional research funds, evaluating for promotions or recruitment particular indices as mentioned above, are often referenced to prioritize distribution in an objective manner. As a result, it becomes necessary to evaluate researchers in various fields. However, each field has its own unique academic characteristics and culture, as well as its own concept of what constitutes an excellent researcher. Hence, resolving issues of using a limited number of indices becomes impossible. Can there be a universal standard for all fields of research? When limiting to the study of life sciences (medical science) in particular, the academic reputation would result from the research result in solving major problems in the field, or the promise of leading to extensive follow-up studies. However, qualitative evaluation remains irreplaceable for matters of fairness. It may be possible in small fields where homogeneity is possible, but the difficulty become apparent immediately when entering the clinical field, where it will not be possible to overcome the evaluation results where an amalgam of different fields are compared in areas where various sciences are integrated and applied.
Eventually, we are only left with resolving to use quantitative indicators that apply to all fields. Where limited positions are available, such as applying to a well-known institution or publishing to a large company, such evaluation is only possible where journals compete. While some reference citations or impact factors of publications, no one can argue that these numbers can replace academic reputation. Often we happen upon news of a journal achieving some miraculous impact factor. Considering that in fields of applied medicine, where the readership and reference possibilities are limited, we cannot imagine such miracle numbers could be a true representation of academic reputation. The impact factor for the International Neurourology Journal (INJ) this year was 1.726 , a small decrement, but a decrease nonetheless, from 1.739 of last year.

While working on the editorial board, I have prided myself of being in personal contact with scores of researchers in our field. But I confess that I must look back on our short coming, and the editorial board should push forward, while worrying how we could introduce the INJ to more of our excellent researchers. To this end, our impact factor may be sacrificed to the point where it may not properly represent our academic reputation.

- Conflict of Interest: No potential conflict of interest relevant to this article was reported. 